\title{
Infinite Number of Twin Primes
}

\author{
S. N. Baibekov, A. A. Durmagambetov \\ L.N. Gumilyov Eurasian National University, Astana, Kazakhstan \\ Email: baibekovsn@mail.ru
}

How to cite this paper: Baibekov, S.N. and Durmagambetov, A.A. (2016) Infinite Number of Twin Primes. Advances in Pure Mathematics, 6, 954-971. http://dx.doi.org/10.4236/apm.2016.613073

Received: October 6, 2016

Accepted: December 12, 2016

Published: December 15, 2016

Copyright $\odot 2016$ by authors and Scientific Research Publishing Inc. This work is licensed under the Creative Commons Attribution International License (CC BY 4.0).

http://creativecommons.org/licenses/by/4.0/

\begin{abstract}
This work is devoted to the theory of prime numbers. Firstly it introduced the concept of matrix primes, which can help to generate a sequence of prime numbers. Then it proposed a number of theorems, which together with theorem of Dirichlet, Siegel and Euler allow to prove the infinity of twin primes.

\section{Keywords}

Prime Numbers, Twin Primes, Composite Numbers, Natural Numbers, Algorithms, Arithmetic Progression, Prime Numbers Matrix, Special Factorial, Generation of Prime Numbers
\end{abstract}

\section{Introduction}

A problem of twin prime numbers infinity formulated at the $5^{\text {th }}$ International Mathematical Congress is one of the main problems of the theory of prime numbers that has not been solved for 2000 years. It has been known that twin prime numbers are pairs of prime numbers which differ from each other by 2. For example, numbers 11 and 13, and numbers 17 and 19 are twin primes, but next adjacent prime numbers 37 and 41 are not twin primes. This problem is also known as the second Landau's problem.

In the year 2013 American mathematician Zhang Yitang from the University of New Hampshire has proved that there are an infinite number of pairs of prime numbers, separated by a fixed distance is greater than 2 but less than 70 million. That is, the number of pairs of twin primes $\left(p_{i}, p_{i+1}=p_{i}+n\right)$ is infinite, where $n$ is greater than 2 but less than or equal to 70,000,000. James Maynard later improved this result to 600 . In 2014, scientists led by Terence Tao (Polymath project) this result was improved up to 246 [1].

A goal of this paper is to prove infinity of twin primes.

To solve this problem, we have proposed a new method, which allows to empirically estimate infinity of twin primes [2]. In this paper we present another simple proof, 
which provides more correct results for twin primes infinity.

First, for convenience we introduce the following notation. As is known, a sequential multiplication of all integers up to a certain number $n$ is called as factorial: $\prod_{i=1}^{n} i=n$ ! Hereafter a sequential multiplication of prime numbers will be occurred frequently, therefore for such cases we use the following notation:

$$
2 * 3 * 5 * 7 * 11 * \cdots * p_{n}=\prod_{i=1}^{n} p_{i}=p_{n} !^{\prime}
$$

Here $p_{i}$ is a prime number with index number $i$. A combination of symbols $p_{n}$ !' means a sequential multiplication of prime numbers from 2 to $p_{n}$ only. We shall call it as a special factorial of a prime number $p_{n}$. For example, $p_{4} !$ is a special factorial of prime number $p_{4}=7$ or $p_{4} !^{\prime}=7 !^{\prime}=2 * 3 * 5 * 7=210$.

\section{Matrices of Prime Numbers}

In this paper we try to prove the infinity of number of twin primes. The proof will be on the basis of properties matrix of prime numbers.

The development of these matrices is implemented as follows.

Let we represent a set of natural numbers in a form of $\boldsymbol{A}_{k}$ matrices family with elements $a(k, I, j)$, where $i$ is a row index number, $j$ is a column index number, and $k$ is an indexing number of matrix $\boldsymbol{A}_{k}$.

Here, a maximum number of rows of matrix $\boldsymbol{A}_{k}$ must be equal to the special factorial $p_{k} !^{\prime}$ i.e. $i_{k, \max }=p_{k} !^{\prime}$. This means, that for every matrix with index number $k$ there is a specific set of prime number sequence: $p_{1}, p_{2}, p_{3}, \cdots, p_{k}$ (note that the last prime number, which corresponds to this matrix is $p_{k}$ ). A number of columns can be arbitrarily large up to infinity.

Here and further it is supposed that we don't know any prime number. Prime numbers will be generated in the course of creating matrices $\boldsymbol{A}_{k}$.

First, we show how matrix $A_{1}$ is formed. For this, we consider a series of natural numbers from 1 to infinity (Figure $1, A_{0}$ ). In this series, number 1 is followed by number 2 . So number 2 is divided by 1 and 2 only. Therefore, the first prime number is 2, i.e. $p_{1}=2$. Then, the first matrix $A_{1}$ built with consideration of the first prime number, has only 2 rows $\left(i_{k, \max }=i_{1, \max }=p_{1} !^{\prime}=2 !^{\prime}=2\right)$. A number of columns is infinite (Figure 1, $\boldsymbol{A}_{1}-\mathrm{a}$ ). Numbers $2,4,6, \ldots$ are located in the first row of matrix $\boldsymbol{A}_{1}$. These numbers form an arithmetic progression. The first term and common difference (step) of this progression are equal to 2, i.e. terms of the first row are respectively equal to:

$$
a(k, i, j)=a(1,1, j)=2+2 *(j-1) \text { where } j=1,2,3, \cdots
$$

The numbers located in the second row of new matrix, also form an arithmetic progression. The first term and common difference of this progression are 3 and 2 respectively, i.e.

$$
a(k, i, j)=a(1,2, j)=3+2 *(j-1) \text { where } j=1,2,3, \cdots
$$




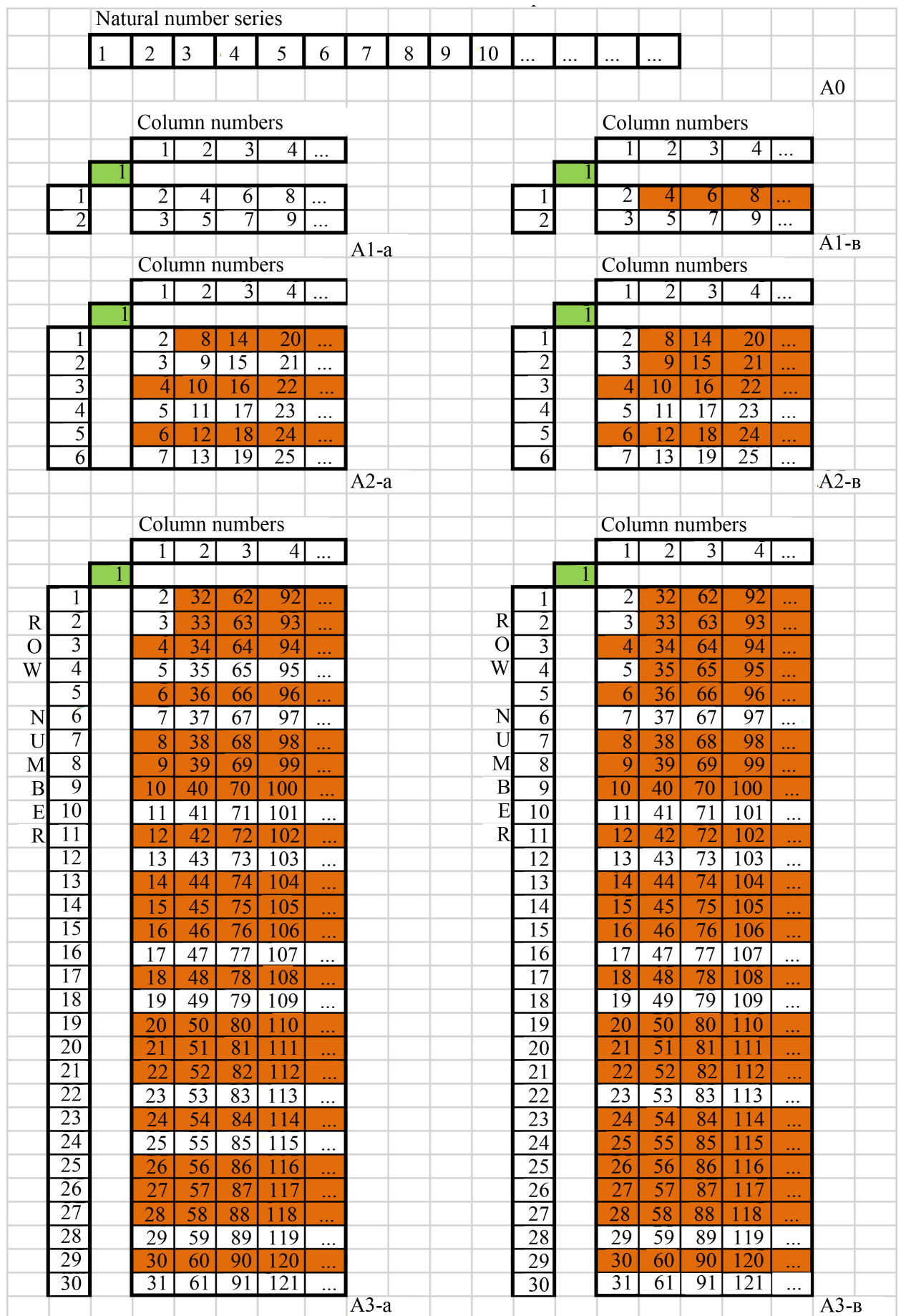

Figure 1. Matrices of prime numbers.

As previously defined, number 2 is a prime number. Therefore, all numbers divisible by 2 are composite numbers. In view of this, all numbers, except 2 , which are located in the first row of the considered matrix (Figure 1, $A_{1}-B$ ) are dark painted for illustrative purposes. Thus, all composite numbers which should be divisible by 2 are defined by using matrix $\boldsymbol{A}_{1}$. 
This implies that number 1 is not a prime number, otherwise all numbers divisible by 1 would be composite numbers. Number 1 is also not a composite number, since it is not divided by other numbers. That is why number 1 is located separately in the upper left corner in this and other matrices.

\section{Algorithm for Matrix Transformation from One to Another Type}

It is seen from matrix $\boldsymbol{A}_{1}$ (Figure 1, $\boldsymbol{A}_{1}-\mathrm{B}$ ), that not painted number next to number 2 is number 3 and it is not divisible by 2 , and therefore it is the second prime number, i.e. $p_{2}=3$.

Thus, we transform matrix $\boldsymbol{A}_{1}$ (Figure 1, $\boldsymbol{A}_{1}-\mathrm{B}$ ) into next matrix $\boldsymbol{A}_{2}$ (Figure 1, $A_{2}$-a). A maximum number of rows of this matrix must be equal to a special factorial of the second prime number $p_{2}=3$, i.e. $i_{k, \max }=i_{2, \max }=p_{2} !^{\prime}=3 !^{\prime}=6$. A number of columns as in the first case can be arbitrary.

For transforming a matrix from one into another type a simple method is used. Implementation of this method lies in a simple transposition of numbers of certain rows and columns of the original matrix into corresponding rows and columns of a new matrix. For example, for forming a first column of matrix $\boldsymbol{A}_{2}$ firstly the numbers 2 and 3 located in the first column of the original matrix $A_{1}$ are transposed to the first and second rows of the new matrix $\boldsymbol{A}_{2}$, then the numbers 4 and 5 of the first matrix are transposed to the third and fourth rows of the new matrix. Then numbers 6 and 7 are also transposed to the fifth and sixth rows of the new matrix. This completes formation of the first column of matrix $\boldsymbol{A}_{2}$. To form a second column of matrix $\boldsymbol{A}_{2}$, we similarly transpose the numbers ( 8 and 9 ), (10 and 11), and (12 and 13) in pairs into a second column of matrix $\boldsymbol{A}_{2}$. Next, we form other columns in a similar way.

Note, that in the new matrix (Figure 1, $\left.A_{2}-a\right)$ all numbers located in the third and fifth rows are dark painted as they, due to matrix $\boldsymbol{A}_{1}$, have been already defined as composite numbers.

In the new matrix all numbers located in each row, as in the case of matrix $\boldsymbol{A}_{1}$, form an arithmetic progression, which in general takes the following form:

$$
a(k, i, j)=(i+1)+p_{k} !^{\prime}(j-1) \text { where } j=1,2, \cdots, \infty ; i=1,2, \cdots, p_{k} !^{\prime}
$$

The common difference of this arithmetic progression is equal to $p_{k} !$. Expression (1) for matrix $\boldsymbol{A}_{2}$, particularly for its second row, appears as follows:

$$
a(2,2, j)=3+6(j-1) \text {, where } j=1,2, \cdots, \infty ; i=1,2, \cdots, p_{2} \text { !' }
$$

As you can see, all the numbers of this row are divisible by 3 . Therefore, they (except number 3 ) are composite numbers. In a view of this they are dark painted in matrix $\boldsymbol{A}_{2}$ (Figure 1, $\boldsymbol{A}_{2}-B$ ). In regard to the numbers located in the fifth row, they are also divided by 3 . However, these numbers, as mentioned above while considering matrix $\boldsymbol{A}_{1}$, have been already defined as composite numbers. A set of numbers of fourth row (and sixth row as well) also form an arithmetic progression. But among them there are both prime and composite numbers. Therefore, numbers of these rows are not painted 
yet. Note, that rows containing only composite numbers are dark painted.

It should be pointed out that here and in all next figures, letter a denotes those matrices (e.g. $\left.A_{1}-a, A_{2}-a, A_{3}-a\right)$, which are formed after transformation of the previous matrix. Letter $B$ denotes those matrices (e.g., $A_{1}-B, A_{2}-B, A_{3}-B$ ), which are obtained after processing has already transformed matrix.

It should be noted that during the process of the second matrix transformation, all numbers divisible by 3 , are finally determined and dark painted accordingly.

It is seen from matrix $\boldsymbol{A}_{2}$ (Figure $1, \boldsymbol{A}_{2}-B$ ) that next to numbers 2 and 3 unpainted number is 5 . Thus it is third prime number $p_{3}=5$. Therefore, the second matrix (Figure 1, $\boldsymbol{A}_{2}-B$ ) is transformed into third matrix $\boldsymbol{A}_{3}$ (Figure 1, $\boldsymbol{A}_{3}$-a). For this we use a similar procedure which was applied for transforming matrix $\boldsymbol{A}_{1}$ into matrix $\boldsymbol{A}_{2}$.

For example, for forming the first column of matrix $\boldsymbol{A}_{3}$ at first the numbers (2 - 7) located in the first column of the original matrix $A_{2}$ are transposed into $\left(1^{\text {st }}-6^{\text {th }}\right)$ row of new matrix $\boldsymbol{A}_{3}$. Then, the numbers (8 - 13) of the second column of matrix $\boldsymbol{A}_{2}$ are transposed into $\left(7^{\text {th }}-12^{\text {th }}\right)$ row of a new matrix. After this, the numbers $(14 \div 19)$ are transposed into $\left(13^{\text {th }}-18^{\text {th }}\right)$ row of the new matrix. Then the numbers $(20 \div 25)$ are transposed into $\left(19^{\text {th }}-24^{\text {th }}\right)$ row of the new matrix and finally, the numbers $(26 \div 31)$ are transposed into $\left(25^{\text {th }}-30^{\text {th }}\right)$ row of the new matrix. This completes formation of the first column of matrix $\boldsymbol{A}_{3}$.

For forming a second column of matrix $\boldsymbol{A}_{3}$, the numbers (32 - 37), (38 - 43), (44 49), (50 - 55) and (56 - 61), located in matrix $\boldsymbol{A}_{2}$, are gradually transposed into second column of matrix $\boldsymbol{A}_{3}$ in a similar way. After that, other columns are similarly formed.

A maximum number of rows in third matrix should be equal to a special factorial of the third prime number $p_{3}=3$, i.e.

$$
i_{k, \max }=i_{3, \max }=p_{3} !^{\prime}=5 !^{\prime}=30 .
$$

For matrix $\quad \boldsymbol{A}_{3}$ expression (1) appears as follows:

$$
a(3, i, j)=(i+1)+30(j-1) \text {, where } j=1,2, \cdots, \infty ; i=1,2, \cdots, p_{3} !^{\prime}
$$

From this expression, we obtain that all the numbers located in the fourth row are divisible by 5 , and those numbers that located in the $24^{\text {th }}$ row are also divisible by 5 . In this context, all of them are accordingly transposed into the series of composite numbers and repainted into dark color (except a prime number 5). Here, in the case of the third matrix (Figure 1, $A_{3}-B$ ) it should be also noted that all numbers which must be divisible by 5 , are finally defined and repainted into dark color (for example, a row with an index number 24).

Note that in all cases, there is no any strict regularity for location of painted and unpainted numbers within one column of any considered matrix. But a picture of mutual arrangement of these numbers within one column is repeated with perfect precision in the next columns (starting from the second column). This regularity of repeating pictures by columns is appeared when each previous matrix $\boldsymbol{A}_{n}$ with a number of rows equal to $p_{n} !$ is transformed into next matrix $A_{n+1}$ with a number of rows equal to 
$p_{n+1} !$.

In matrix $\boldsymbol{A}_{3}$ (Figure 1, $\boldsymbol{A}_{3}-B$ ) a number 7 which is next to the numbers 2, 3, and 5 is not painted. Therefore, it is a fourth prime number $p_{4}=7$. Now, knowing the fourth prime number 7, matrix $\boldsymbol{A}_{3}$ can be similarly transformed into the next fourth matrix $\boldsymbol{A}_{4}$. In this matrix a maximum number of rows must be equal to a special factorial of prime number 7 , i.e. $7 !=210$.

In this case, carrying out a number of similar operations, as in previous cases, we can finally identify a set of all composite numbers, which should be divisible by 7 . Similarly, we can build other matrices.

Here we have presented a procedure that allows to perform mechanical transformation of prime numbers matrices from one type to another. In general case an algorithm of this transformation is as follows.

Let there be given matrix $\boldsymbol{A}_{k}$ with elements $a(k, i, j)$, where $i$ is an row index number, $j$ is a column index number, and $k$ is an index number of matrix $\boldsymbol{A}_{k}$. Then, as it follows from (1), the elements of this matrix are determined by the following expression:

$$
a(k, i, j)=(i+1)+p_{k} !^{\prime}(j-1) \text {, where } i=1,2, \cdots, p_{k} !^{\prime}, j=1,2, \cdots, \infty .
$$

And a maximum number of matrix $\boldsymbol{A}_{k}$ rows should be equal to a special factorial $p_{k} !$, i.e. $i_{k, \max }=p_{k}$ !'. A number of columns can be arbitrary large up to infinity. On the other hand, a family of numbers located in any selected row of this matrix, creates an arithmetic progression the first term of which is equal to $(i+1)$ and common difference of the progression is equal to $p_{k} !$.

Then the algorithm of building next matrix $\boldsymbol{A}_{k+1}$ lies in a simple calculation of values of new matrix elements using the following equation:

$$
a(k+1, i, j)=(i+1)+p_{k+1} !^{\prime}(j-1) \text {, where } i=1,2, \cdots, p_{k+1} !^{\prime}, j=1,2, \cdots, \infty .
$$

Again, in this case a maximum number of matrix $A_{k+1}$ rows should be also equal to a special factorial $p_{k+1} !^{\prime}$, i.e. $i_{k+1, \max }=p_{k+1} !^{\prime}$. A number of columns can be also arbitrary large up to infinity as in case of matrix $\boldsymbol{A}_{k}$. In this case a family of numbers located in any selected row of this matrix, also creates an arithmetic progression the first term of which is equal to $(i+1)$ while common difference of the progression is equal to $p_{k+1} !^{\prime}$.

In a similar way matrix $\boldsymbol{A}_{k+1}$ is being transformed into matrix $\boldsymbol{A}_{k+2}$, etc.

Here, based on the Dirichlet's theorem on prime numbers in arithmetic progressions, it follows that if the first term and difference of the progression are not coprime numbers, then this progression will not contain any prime number or will contain only one prime number. And this prime number is the first term of the progression.

It also follows from the Dirichlet's theorem that if the first term and difference of the progression are coprime numbers, then this progression contains prime numbers and composite numbers as well.

Therefore, in our case, first we determine if the first term and difference of the progression that consists of the numbers located in considered row of given matrix are coprime numbers. If they are not coprime numbers, then we conclude that all numbers of 
this row are composite numbers and they are dark painted for illustration purposes.

If the first term and difference of the considered progression are coprime numbers, then as mentioned above, this row contains both prime and composite numbers. Therefore, the numbers of these rows remain unpainted. Note, that dark painted are only those rows that contain only composite numbers.

Now, using matrices $\boldsymbol{A}_{k}$, we will try to determine a number of twin primes.

\section{Infinite Number of Twin Primes}

First, we set a number of definitions:

Definition 1. If in a certain row of a matrix there are only composite numbers, then the row is dark painted for illustration purposes and for convenience we call it as a painted row.

Definition 2. If in a certain row of a matrix there are both prime and composite numbers, the row is not painted and for convenience we call such rows as not painted row.

Definition 3. If the first number of a row is not painted but the rest numbers are painted, then this not painted number is a prime number and the rest numbers are composite.

Definition 4. If a difference between index numbers of two neighbor and not painted rows is equal to 2 , then such rows we call a pair of twin rows or twin rows. For the numbers located in different rows but in one column of twin rows pairs, an equation $|a(k, i, j)-a(k, i \mp 2, j)|=2$ is always satisfied.

Definition 5. If an index number of a certain painted row differs from an index number of the nearest not painted row by greater than 2 , then the raw is called as a single row.

From these definitions it follows that twin prime numbers can be onlyin twin rows.

A goal of the paper is determine a total number of prime numbers. Therefore, hereafter we will put main emphasis on pairs of twin rows.

Theorem 1. A number of twin rows pairs in matrix $\boldsymbol{A}_{k}$ is monotonically increased with a growth of index number $k$ of the matrix and also in each row of any twin rowspair there are an infinite number of prime numbers.

As is known, all twin prime numbers can be located in paired twin rows only. Moreover, if at some point, for example when considering matrix $\boldsymbol{A}_{k}$, all pairs of twin rows are disappeared, then it obviously that they will not appear in next matrices. In that case, it means that a number of twin prime numbers should be limited.

We will analyze whether such case is possible and prove Theorem 1 conjointly.

\section{Proof of Theorem 1.}

Let suppose that some matrix has only one single pair of twin rows (for example, as in the case of Figure 1, $\left.\boldsymbol{A}_{2}-B\right)$. Here, there is reason to assume that in the course of further transformation of this matrix into the next matrices, pairs of twin rows may disappear. But, in fact the opposite is true. When transforming the matrix into the next matrix a number of twin rows pairs, as shown above, becomes larger. 
For example, in matrix $\boldsymbol{A}_{2}$ there is only one single pair of twin rows (Figure 1, $\boldsymbol{A}_{2}$ $-B)$. From the expression (1) it follows that terms of the arithmetic progression, which are located in the rows of this single pair of twin rows are defined by the expression:

$$
(6 \mp 1)+6(j-1)=\left(p_{2} !^{\prime} \mp 1\right)+p_{2} !^{\prime}(j-1) \text { where } j=1,2, \cdots, \infty
$$

Here signs "-" and "+" correspond to upper and lower row of the pair of twin rows respectively.

But this only one pair of rows generates $5\left(p_{3}=5\right)$ new pairs of rows during transformation of this matrix into matrix $\boldsymbol{A}_{3}$. That is, the original unique pair of twin rows is ungrouped by 5 new pairs of rows.

A set of numbers located in each row of 5 new pairs of rows of matrix $\boldsymbol{A}_{3}$ also forms an arithmetic progression with a constant $p_{3} !^{\prime}=30$ and is defined by the expression:

$$
(6 m \mp 1)+30(j-1)=\left(p_{2} !^{\prime} m \mp 1\right)+p_{3} !^{\prime}(j-1),
$$

where $j=1,2,3, \cdots, \infty ; m=1,2, \cdots, p_{3}$.

From expression (3) we obtain that if at some value of $m=1,2, \cdots, p_{3}$ the following equation is satisfied

$$
\frac{p_{2} !^{\prime} m \mp 1}{p_{3}}=\text { int eger, }
$$

then all numbers of this row are divided by $p_{3}$ exactly. Therefore, the numbers are composite. In fact, it is known that within interval of $00<m<p_{3}$ the Equation (4) with regard to the parameter $m$ has unique solution [3] [4] and [5]. For example, equation (4) for the case of $\left(p_{2} !^{\prime} m-1\right)$ is satisfied at $m=1$ and for the case of $\left(p_{2} !^{\prime} m+1\right)$ at $m=4$. That is, at $m=1$ and $m=4$ a pair of rows in question is not a pair of twin rows and corresponding row for which the equation (4) is satisfied, is dark repainted. As a result only 3 of 5 newly formed pairs of rows are twin rows.

All the numbers of each row of 3 newly formed pairs of twin rows, as stated above, form an arithmetic progression and in each of them the first term and difference of the arithmetic progression are coprimes, i.e.:

$$
\left(p_{2} !^{\prime} m \mp 1, p_{3} !^{\prime}\right) \equiv 1 \text {, where } m=2,3 \text { and } 5, m \neq 1, m \neq 4
$$

In virtue of this, it follows from Dirichlet theorem on prime numbers in arithmetic progressions, that in each row of these three pairs of twin rows there is an infinite number of prime numbers.

Now we consider a transformation of matrix $\boldsymbol{A}_{3}$ into matrix $\boldsymbol{A}_{4}$. In this case, each pair of twin rows of matrix $\boldsymbol{A}_{3}$ generates $7\left(p_{4}=7\right)$ new pairs of rows and totally 21 new pairs of rows are formed in new matrix $\boldsymbol{A}_{4}$. Values of numbers located in the rows of these pairs are defined by the expression:

$$
\left[p_{2} !^{\prime} p_{i}+p_{3} !^{\prime}(m-1) \mp 1\right]+p_{4} !^{\prime}(j-1)
$$

where $j=1,2,3, \cdots, \infty ; m=1,2, \cdots, p_{4} ; i=1,2,3$.

It can be seen from (5), that a set of numbers lying in each row of newly created 21 
pairs of rows, separately forms an arithmetic progression with the difference of $p_{4} !^{\prime}$ and the first terms defined as $\left[p_{2} !^{\prime} p_{i}+p_{3} !^{\prime}(m-1) \mp 1\right]$.

We now consider which of these 21 pairs of rows of matrix $\boldsymbol{A}_{4}$ are pairs of twin rows. For this purpose we analyze divisibility of the first terms of the aforementioned arithmetic progressions by $p_{4}=7$. In addition, for convenience and visualization, we consider a case where $i=3$, but at the same time we mean the cases where $i=1$ and $i=$ 2. Then from (5) we find that values of numbers lying in 7 new rows of matrix $\boldsymbol{A}_{4}$ generated by the last pair of twin rows of matrix $A_{3}$ are determined by the expression: $p_{3} ! m \mp 1+p_{4} !^{\prime}(j-1)$, where $j=1,2,3, \cdots, \infty ; m=1,2, \cdots, p_{4}$.

Let we consider divisibility of the first terms $p_{3} !^{\prime} m \mp 1$ of the arithmetic progression in question by $p_{4}$, i.e. a satisfiability of the equation:

$$
\frac{p_{3} ! m \mp 1}{p_{4}}=\text { int eger }
$$

In this case, in the same way as for case (4), we find that within an interval of $0<m<p_{4}$ this equation with reference to parameter $m$ (for the case of $p_{3} !^{\prime} m-1$, and also for the case of $\left.p_{3} ! m+1\right)$ has an unique solution. Therefore, in this case 2 pairs of the rows in question are no longer pairs of twin rows. If we additionally consider the cases when $i=1$ and $i=2$, then we finally obtain that 6 pairs of rows of 21 newly formed pairs of rows cease to be pairs of twin rows, and corresponding rows, as shown above, are dark repainted. As a result, a number of new pairs of twin rows in matrix $\boldsymbol{A}_{4}$ is equal to 15 .

Besides, all first terms and difference of the arithmetic progression formed from the numbers lying in each row of the newly created 15 pairs of twin rows of matrix $\boldsymbol{A}_{4}$, are coprimes, i.e.:

$$
\left(\left[p_{2} !^{\prime} p_{i}+p_{3} !^{\prime}(m-1) \mp 1\right], p_{4} !^{\prime}\right) \equiv 1,
$$

where $i=1,2,3 ; m \in\left(1, p_{4}\right)$ and $m \neq 5,6,9,10,17,18$.

In view of this, it follows from Dirichlet theorem for prime numbers in arithmetic progression, that in each row of 15 newly formed pairs of twin rows there is an infinite number of prime numbers.

If we consider further similar transformations of matrices into the next following matrices, for example, matrix $\boldsymbol{A}_{k-1}$ into matrix $\boldsymbol{A}_{k}$, then every time we verify that any pair of twin rows of the original matrix generates $p_{k}$ new pairs of rows in new matrix. In addition, 2 pairs of them will not be pairs of twin rows and corresponding rows are moving into a rank of painted and single rows. From this we obtain that in any matrix $\boldsymbol{A}_{k}$ a total number of rows $\left(i_{k \text {, max }}\right)$ and total number of twin row pairs $\left(m_{k}\right)$ are respectively equal to:

$$
i_{k, \text { max }}=i_{k-1, \max } * p_{k} \text { and } m_{k}=m_{k-1}\left(p_{k}-2\right)
$$

or

$$
i_{k, \max }=p_{k} !^{\prime} \text { and } m_{k}=\left(p_{k}-2\right) !^{\prime}, k \geq 2
$$


where-an index number of matrix $\boldsymbol{A}_{k}$ and/or prime number $p_{k}$, and

$$
\left(p_{k}-2\right) !^{\prime}=\left(p_{2}-2\right)\left(p_{3}-2\right) * \ldots *\left(p_{k}-2\right)==1 * 3 * 5 * 9 * 11 * \ldots *\left(p_{k}-2\right)
$$

It follows from (7) that a number of twin rows pairs is monotonically increased while moving to the next matrices, i.e. with increasing of index number $k$ of matrix $\boldsymbol{A}_{k}$,. On the other side, a set of numbers located in each row of these $m_{k}$ pairs of twin rows, forms an arithmetic progression. And the first term and difference of each this progression are coprimes. Therefore it follows from Dirichlet's theorem that in each row of any pair of twin rows there is an infinite number of prime numbers.

\section{The theorem 1 is proved.}

As is shown in (7), a number of twin row pairs will be progressively increasing during the process of moving to the next matrices. But a number of ordinary rows of each next matrix is increased as a special factorial $p_{k} !^{\prime}$. In a view of this, a density of twin row pairs is progressively decreased along with the matrices since the ratio $\frac{\left(p_{k}-2\right) !^{\prime}}{p_{k} !^{\prime}}$ is progressively reduced with rising of $k$.

Theorem 2. There are prime twin numbers in any pair of twin rows of any matrix $A_{k}$.

As shown above, all twin prime numbers can be located in twin rows only. But the question arises are there cases where in some pair of twin rows no any pair of two prime numbers is located in one column. Then, due to the asymmetry (i.e. due to the skewness of prime numbers location) there will be no any pair of prime twin numbers in this pair of twin rows. If such skewness happens in all twin rows pairs of this matrix, then this and all next matrices will no longer contain prime twin numbers. Therefore we can definitely say that a number of prime twins should be limited.

We will analyze this case now and prove the Theorem 2.

\section{Proof of the Theorem 2.}

Let consider matrix $\boldsymbol{A}_{2}$ which contains only one unique pair of twin rows (Figure 1, $\left.\boldsymbol{A}_{2}-B\right)$. On the other hand, as shown above, twin prime numbers can be located in pairs of twin rows only. This means that all existing twin prime numbers are located only in this unique pair of twin rows.

A simple analysis shows that pairs of twin prime numbers conform to some simple rules. In particular, the last digit of any prime number (except 2 and 5) can not be an even number and it can not be equal to 5 as well. This means that last digits of the first and second number of any pair of twins should be respectively (1 and 3) and (7 and 9), and ( 9 and 1 ) as well. Therefore, a set of twin prime numbers should be divided into 3 subsets on these grounds. In fact, when we form matrix $\boldsymbol{A}_{3}$, a single pair of twin rows of matrix $\quad \boldsymbol{A}_{2}$ generates new three pairs of twin rows in matrix $\boldsymbol{A}_{3}$. Moreover, the last digits of each number located in the rows of individually selected pair of twin rows are respectively equal (1 and 3$)$ and (7 and 9) and (9 and 1). This fact is readily illustrated by Figure 1, $\boldsymbol{A}_{3}-B$.

Consequently, the fact that in each pair of twin rows of matrix $\boldsymbol{A}_{3}$ there is a set twin 
primes, which is sorted with regard to a value of the latest digit, is beyond question. That is, in pairs of twin rows of the matrix $\boldsymbol{A}_{3}$ the skewness will not appear.

Now we consider next matrix $\boldsymbol{A}_{4}$. The analysis we performed shows that all members of each pair of twin rows of this matrix are more ordered than in case of matrix $\boldsymbol{A}_{3}$. For example, last two digits of each number of the arithmetic progression, located in any row of any twin row pair of matrix $\boldsymbol{A}_{4}$ form cyclically increasing sequence: 21 , $31,41, \ldots, 81,91,01,11,21,31, \ldots$

Here we suppose that an average distance by columns between cells, where adjacent prime numbers are located in one pair of twin rows of matrix $\boldsymbol{A}_{4}$, must be less than an average distance of cells, where adjacent primes in one pair of twin rows of matrix $\boldsymbol{A}_{3}$ are located.

If this is so, then in each pair of twin rows of matrix $\boldsymbol{A}_{4}$ there inevitably are twin primes. Since in this case, due to the tightness even if above-mentioned skewness of prime numbers appears in matrix $\boldsymbol{A}_{4}$, then it appears in a less degree then in case of matrix $\boldsymbol{A}_{3}$. At least there will not be a general skewness of prime numbers in matrix $A_{4}$.

To verify this, first we enter a new parameter $d_{i, i+1}$ which is a distance by columns between the cells, where adjacent prime numbers with index numbers $i$ and $i+1$ (Figure 2) are located:

$$
d_{i, i+1}=\left|M_{i+1}-M_{i}\right|,
$$

where $M_{i}$ is an index number of the column in which a cell of $i$-th prime number is located. Moreover, if two adjacent prime numbers are located in two mutually adjacent cells along one row (i.e. horizontally, as shown in Figure 2, a), then a distance between these cells is equal to 1 :

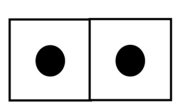

(a)

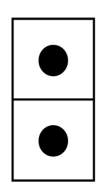

(b)

\begin{tabular}{|l|l|l|l|l|l|l|l|l|l|l|l|l|l|l|}
\hline \multicolumn{11}{|c|}{ Column numbersM } \\
\hline 1 & 2 & 3 & 4 & 5 & 6 & 7 & 8 & 9 & 10 & 11 & $\ldots$ & $k-2$ & $k-1$ & $k$ \\
\hline
\end{tabular}

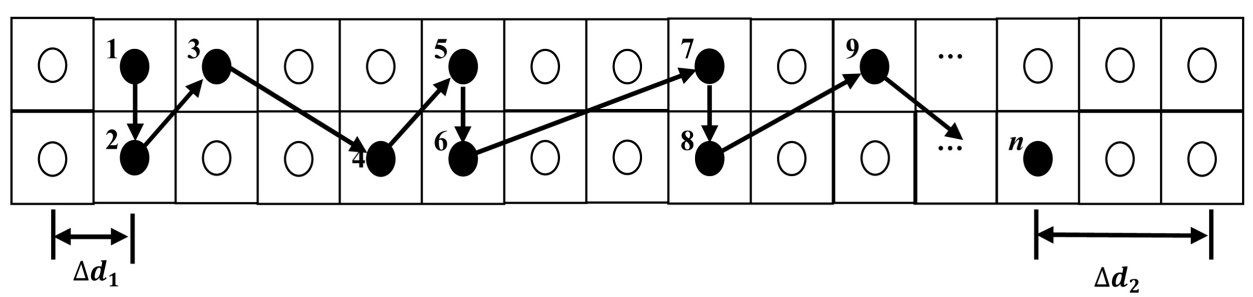

(c)

Figure 2. Location of prime numbers in a pair of twins rows. 


$$
d_{i, i+1}=\left|M_{i+1}-M_{i}\right|=1
$$

On the other hand, if two adjacent prime numbers are located in two neighboring and adjoining cells lying in the same column (i.e. vertically as shown in Figure 2, B), then a distance between these cells is equal to zero:

$$
d_{i, i+1}=\left|M_{i}-M_{i}\right|=0
$$

In this case these two prime numbers are twins.

As an example, we now consider a fragment of one pair of twin rows of any matrix. Let suppose that this fragment contains $n$ prime numbers, as shown in Figure 2(c). In this figure light circles denote cells, in which composite numbers are located and dark circles mark cells with prime numbers. Then, above mentioned average distance by columns $d_{a v}$ between cells, where adjacent prime numbers are located, will be equal to:

$$
d_{a v}=\frac{\sum_{i=1}^{n-1} d_{i, i+1}}{n-1}
$$

In this case, as shown above, a distance by columns between cells, where adjacent prime numbers with index numbers ( 1 and 3 ) and (4 and 6) are located, is equal 1, i.e.: $d_{1,3}=\left|M_{3}-M_{2}\right|=1 ; d_{4,6}=\left|M_{6}-M_{5}\right|=1$.

On the other hand a distance by columns between cells of adjacent prime numbers with index numbers ( 1 and 2$)$, (5 and 6) and (7 and 8) is equal to zero, that is $d_{1,2}=M_{2}-M_{2}=0 ; d_{5,6}=M_{6}-M_{6}=0 ; d_{7,8}=M_{9}-M_{9}=0$. Therefore, if we consider a prime number with index number 1 , then its nearest adjacent prime number on the right side is a prime number with index number 2, rather than a number with index number 3 (Figure 2(c)). If we consider a prime number with index number 4 , its nearest neighbor to the right side is a prime number with index number 5 , rather than a number with index number 6 . This is easily seen, if a difference between values of these numbers is calculated using formula (1). Similarly, it can be easily determined that the nearest neighbor of a prime number with index number 9 on the left side is a prime number with a index number 8 , rather than a number with index number 7 , although these two prime numbers ( 7 and 9 ) are located in the same row.

Note, that the numbers with index numbers ( 1 and 2$)$, (5 and 6), and (7 and 8) are twins (Figure 2(c)).

It should be noted that the first prime number can be located in a cell that is lying not in the first column of the considered fragment (Figure 2(c)). Similarly, we can say that a cell in which the last prime number with index number $n$ is located, may be also situated not in the last column. These cases are not taken into account in (9), parameters $\Delta d_{1}$ and $\Delta d_{2}$ are not presented in expression (9). $\Delta d_{1}$ is a number of columns calculated from the beginning of the considered fragment to a cell containing first prime number and $\Delta d_{2}$ is a number of columns from a cell with last prime number to the end of the considered fragment (Figure 2(c)). To take this into account, we consider a sum:

$$
\Delta d=\Delta d_{1}+\Delta d_{2}
$$


It should be noted that a value of the considered parameter $\Delta d$ is comparable and most likely equal to the distance by columns from the cell of the last prime number with an index number $n$ to the cell, where next prime number with index number $n+1$ will be located. As it follows from Figure 2(c) this number, is located outside the analyzed fragment, but it will be the nearest adjacent prime number from the right side for $n$-th prime number. From this it follows that:

$$
\Delta d \simeq d_{n, n+1}
$$

If we also add the parameter $\Delta d$ into the sum in a numerator of the expression (9), then we exactly obtain "a length" of the considered fragment (Figure 2(c)):

$$
\sum_{i=1}^{n-1} d_{i, i+1}+\Delta d=M_{k}
$$

Here, a number of summands in the numerator of expression (9) will be greater by 1 , i.e. a number of prime numbers in question is supposedly increased by 1 and becomes equal to $n+1$. Accordingly, expression (9) takes the following form:

$$
d_{a v}=\frac{\sum_{i=1}^{n-1} d_{i, i+1}+\Delta d}{(n+1)-1}=\frac{M_{k}}{n}
$$

Now we consider a real case of matrix $A_{k-1}$ fragment with dimensions $\left(m_{k-1}, M_{k-1}\right)$. Here $m_{k-1}$ is a number of pairs of twin rows, $M_{k-1}$ is a number of columns in given fragment of matrix $\boldsymbol{A}_{k-1}$, which corresponds to a prime number $p_{k-1}$. It should be noted here that a value of $M_{k-1}$ should be sufficiently large so it make statistical sense. For example, it should be:

$$
M_{k-1} \gg p_{k} !
$$

Let $\pi_{k-1}$ is a total number of all prime numbers lying in all pairs of twin rows of the considered fragment of matrix $\boldsymbol{A}_{k-1}, \pi_{k-1, l}$ is a number of prime numbers contained in one selected pair of twin rows with index number 1 . In addition a total number of twin rows pairs should be equal $m_{k-1}$, i.e. $l$ takes values from 1 to $m_{k-1}$, in short $l=1 \div m_{k-1}$

Then when applying (10) for the case of matrix $\boldsymbol{A}_{k-1}$ we obtain:

$$
d_{k-1, l}=\frac{M_{k-1}}{\pi_{k-1, l}}
$$

where $d_{k-1, l}$ is an average distance by columns between cells where adjacent prime numbers, lying in one pair of twin rows with index number $l$, are located.

Note that here and further the first index of the parameter in question (in this case index $k-1$ ) will correspond to index number of the considered matrix, and second index of this parameter (in this case index $l$ ) is an index number of the analyzed pair of twin rows.

Above we made an assumption that prime numbers in pairs of twin rows of each new matrix must be spaced more closely than in pairs of twin rows of previous matrix. To analyze and evaluate the assumption we will analyze a value of $d_{k-1, l}$ by pairs of twin 
rows of the considered fragment.

From the papers of Siegel [6] [7] [8] and other researchers [9] [10] [11] [12] it follows that if the constants (common differences) of different arithmetic progressions are equal to each other and the first term and common difference of each arithmetic progressions are co-primes, then prime numbers are distributed similarly and identically in these progressions. On the other hand, as shown above, a sequence of numbers located in any row of any pair of twin rows of matrix $\boldsymbol{A}_{k}$ forms an arithmetic progression with the same common difference equal to $p_{k} !^{\prime}$. In addition the first term and common difference of these arithmetic progressions are co-primes. These progressions differ from each other only by a value of the first term and they are identical in all other respects. This means that within the considered fragment quantities of prime numbers in any pair of twin rows are approximately equal, i.e.:

$$
\pi_{k-1, a v} \simeq \pi_{k-1,1} \simeq \pi_{k-1,2} \simeq \cdots \simeq \pi_{k-1, l} \simeq \cdots \simeq \pi_{k-1, m_{k-1}},
$$

where $\pi_{k-1, a v}$ is an average quantity of prime numbers containing in a pair of twin rows of the considered fragment of matrix $\boldsymbol{A}_{k-1}$.

Then from this and expression (12) we obtain:

$$
d_{k-1, a v} \simeq d_{k-1,1} \simeq d_{k-1,2} \simeq \cdots \simeq d_{k-1, l} \simeq \cdots \simeq d_{k-1, m_{k-1}},
$$

or

$$
d_{k-1, a v}=\frac{M_{k-1}}{\pi_{k-1, c p}}
$$

where $d_{k-1, a v}$-an average distance by columns between cells where adjacent prime numbers, lying in a pair of twin rows of the considered fragment of matrix $\boldsymbol{A}_{k-1}$, are located.

On the other side, it follows from (13) that

$$
\pi_{k-1, a v}=\frac{\sum_{j=1}^{m_{k-1}} \pi_{k-1, j}}{m_{k-1}}=\frac{\pi_{k-1}}{m_{k-1}}
$$

Here, we call attention to the following:

1) A certain selected row of any pair of twin rows in any matrix, excepting matrices $\boldsymbol{A}_{0}$ and $\boldsymbol{A}_{1}$ (Figure 1, $\boldsymbol{A}_{0}$ and Figure 1, $\boldsymbol{A}_{1}$ ), could not contain prime twin numbers as shown above.

2) If two prime numbers, as shown above, are located in one column within one pair of twin rows, then they are twins. In this case a distance by columns between cells, where these prime twin numbers are located should be equal to zero.

3) Considered distance $d_{k, a v}$ by columns between cells, where adjacent prime numbers are located, should not be confused with a difference, i.e. with a distance between values of adjacent prime numbers. It is plain, that in one cell of considered fragment of any matrix only one number can be located. In addition, difference of values of adjacent prime numbers, which are located in different, but adjacent to each other by row, cells of the fragment can take a value of any even integer.

As shown in a case of proving Theorem 1, it follows from (7), that while transform- 
ing matrix $\boldsymbol{A}_{k-1}$ into matrix $\boldsymbol{A}_{k}$, each pair of twin rows of matrix $\boldsymbol{A}_{k-1}$ generates $p_{k}-2$ new pairs of twin rows and 2 additional single rows in new matrix. As a result, each pair of twin rows of matrix $\boldsymbol{A}_{k-1}$ creates $p_{k}-1$ pairs of new rows. Totally, $m_{k-1}$ pairs of twin rows generate $m_{k}=m_{k-1}\left(p_{k}-2\right)$ new pairs of twin rows and additionally $m_{k}^{\prime}=m_{k-1}$ pairs of single rows in new matrix $\boldsymbol{A}_{k}$.

In brief, a set of $\pi_{k-1}=m_{k-1} M_{k-1} / d_{k-1, c p}$ prime numbers lying in all pairs of twin rows of the considered fragment of matrix $\boldsymbol{A}_{k-1}$ is redistributed by $m_{k}^{\prime}=m_{k-1}\left(p_{k}-1\right)$ new pairs of rows of new fragment corresponding to new matrix $\boldsymbol{A}_{k}$. Then an amount of prime numbers located in all newly created $m_{k}$ pairs of twin rows of matrix $\boldsymbol{A}_{k}$ fragment is defined by the expression:

$$
\pi_{k}=\frac{m_{k} \pi_{k-1}}{m_{k}+m_{k}^{\prime}}=\frac{m_{k} \pi_{k-1}}{m_{k-1}\left(p_{k}-1\right)}
$$

Therefore, a number of prime numbers, located in one selected pair of twin rows of the considered fragment of new matrix $\boldsymbol{A}_{k}$, is on the average:

$$
\pi_{k, a v}=\frac{\pi_{k}}{m_{k}}=\frac{\pi_{k-1}}{m_{k-1}\left(p_{k}-1\right)}
$$

On other hand, while transforming a fragment of $\boldsymbol{A}_{k-1}$ matrix into a fragment of matrix $\boldsymbol{A}_{k}$, a number of columns in new fragment, as shown above, is reduced by $p_{k}$ times, i.e. $M_{k}=M_{k-1} / p_{k}$.

Then from (14), (15) and (16) we obtain $d_{k, a v}$, an average distance by columns between the cells, where adjacent prime numbers, lying in one pair of twin rows of the considered fragment of matrix $\boldsymbol{A}_{k}$, are located

$$
d_{k, a v}=\frac{M_{k}}{\pi_{k, a v}}=\frac{m_{k-1} M_{k-1}}{\pi_{k-1}} * \frac{p_{k}-1}{p_{k}}=d_{k-1, a v} \frac{p_{k}-1}{p_{k}}
$$

Here we note the following. In the numerator (10) parameter $\Delta d$ is treated as a single term. In fact this option consists of two settings $\Delta d_{1}$ and $\Delta d_{2}$. So if these two settings in the numerator (10) will be counted simultaneously, then the number of summands in the numerator (10) will be equal to $n+2$. With this in mind, the expression (10) takes the following form:

$$
d_{a v}=\frac{\sum_{i=1}^{n-1} d_{i, i+1}+\Delta d_{1}+\Delta d_{2}}{(n+2)-1}=\frac{M_{k}}{n+1}
$$

If using this expression, enter appropriate simple changes to (12) and (14), then the expression (17) eventually goes into the following form:

$$
d_{k, a v}=\frac{M_{k}}{\pi_{k, a v}+1}=\frac{1}{\frac{1}{d_{k-1, a v}}+\frac{p_{k}-1}{M_{k-1}}} * \frac{\left(p_{k}-1\right)}{p_{k}}
$$

From the inequality (11) get that

$$
\frac{p_{k}-1}{M_{k-1}}=0
$$


Therefore, with this in mind, we obtain that the expression for the parameter $d_{k, a v}$ will have the same appearance-what is in (17). In short, the joint consideration of parameters $\Delta d_{1}$ and $\Delta d_{2}$ gives the same result, which is obtained at considering only one parameter $\Delta d$.

From this it follows that with increase of index number $k$ of matrix $\boldsymbol{A}_{k}$ an average distance $d_{k, a v}$ between the cells of adjacent prime numbers in any pair of twin rows of matrix $\boldsymbol{A}_{k}$ decreases continuously.

As can be seen, "a density", i.e. closeness of prime numbers is increasing. Therefore due to infinity of prime numbers and identity of their distribution in any pair of twin rows of matrix $\boldsymbol{A}_{k}$, a probability of occurrence of twin numbers will be greater than in case of previous matrix.

In particular, due to the fact that general skewness of prime numbers in pairs of twin rows of matrix $\boldsymbol{A}_{3}$ does not exist, as shown above, then it will not appear in pairs of twin rows of the next matrices $\boldsymbol{A}_{4}, \boldsymbol{A}_{5}, \boldsymbol{A}_{6}, \cdots, \boldsymbol{A}_{k}, \cdots$ So, there are twin prime numbers in each pair of twin rows of any matrix $\boldsymbol{A}_{k}$.

The Theorem 2 is proved.

Now we consider a problem posed in front of this paper.

Theorem 3. A number of twin primes is infinite.

\section{Proof of the Theorem 3.}

As is shown above, each matrix $\boldsymbol{A}_{k}$ corresponds to a certain prime number $p_{k}$. It is known that a number of prime numbers is infinite. Therefore, a number of matrix $\boldsymbol{A}_{k}$ variations is also infinite. On the other hand, twin prime numbers can be located in pairs of twin rows only.

It follows from the Theorem 1 and expression (7) that with rising growth of matrix ${ }_{k}$ index number, a number of pairs of twin rows in this matrix is steadily increased. i.e.

$$
\lim _{k \rightarrow \infty} m_{k}=\lim _{k \rightarrow \infty}\left(p_{k}-2\right) !^{\prime}=\infty
$$

It also follows from the Theorem 2, that in any pair of twin rows there are prime twin numbers. This entire means that a number of prime twins is infinite.

This conclusion is also unavoidably followed from the expression (17).

If in (17) we express the parameter $d_{k-1, a v}$ in terms of $d_{k-2, a v}$, which is a mean distance by columns between cells, where adjacent prime numbers are located in a pair of matrix $A_{k-2}$ twin rows, then we obtain

$$
d_{k-1, a v}=d_{k-2, a v} \frac{p_{k-1}-1}{p_{k-1}}
$$

then

$$
d_{k, a v}=d_{k-2, a v} \frac{p_{k-1}-1}{p_{k-1}} * \frac{p_{k}-1}{p_{k}}
$$

Next, in the same manner, we transform parameter $d_{k-2, a v}$ through use of $d_{k-3 a v p}$, then $d_{k-3, a v}$ through use of $d_{k-4, a v}$, etc. While continuing the transformation up to $d_{1, a v}$, we obtain that an average distance by columns between cells of prime numbers in 
any pair of matrix $\boldsymbol{A}_{k}$ twin rows can be generally determined by the expression:

$$
d_{k, a v}=d_{1, a v} \frac{\left(p_{1}-1\right)\left(p_{2}-1\right) \cdots\left(p_{k}-1\right)}{p_{1} p_{2} * \cdots * p_{k}}=d_{1, a v} \frac{\left(p_{k}-1\right) !}{p_{k} !}
$$

where $k \geq 2$ and $d_{1, a v}$ is a mean distance between cells of adjacent prime numbers lying in a single not painted row of matrix $\boldsymbol{A}_{1}$ (Figure 1, $\boldsymbol{A}_{1}-B$ ).

It follows from (18), that with rising of index number $k$ of matrix $\boldsymbol{A}_{k}$ an average distance $d_{k, a v}$ between cells of prime numbers in any pair of twin rows of matrix $\boldsymbol{A}_{k}$ is progressively decreasing. And if $k \rightarrow \infty$ we obtain that $d_{k, a v} \rightarrow 0$. In fact, having done small transformation we obtain from (18) that

$$
\ln d_{k, a v}=\ln d_{1, a v}-\left(\sum_{i=1}^{k} \frac{1}{p_{i}}+\sum_{i=1}^{k} \sum_{m=2}^{\infty} \frac{1}{m * p_{i}^{m}}\right)
$$

Here, the infinite series containing reciprocals of prime numbers diverges, as was shown by Euler [13], i.e.:

$$
\sum_{i=1}^{\infty} \frac{1}{p_{i}}=\infty
$$

Therefore,

$$
\lim _{k \rightarrow \infty} d_{k, a v}=0
$$

This means that with a growth of matrix $\boldsymbol{A}_{k}$ 's index number an average distance $d_{k, a v}$ between the cells, where adjacent primes are located, tends to zero. In addition the infinity of twins is not only inevitable but also obvious because it follows from (7) that at $k \rightarrow \infty$ a number of twin rows pairs tends to infinity: $\lim _{k \rightarrow \infty} m_{k}=\infty$. On the other hand as it follows from the Theorem 2, in each of these pairs of twin rows there are twin prime numbers.

The Theorem 3 is proved.

\section{Conclusion}

Study authors introduce the concept of matrix primes for researching of the properties of prime numbers. After, a number of theorems were proved in the work. Using these theorems and the theorems of Dirichlet, Siegel and Euler the proof of the infinity of twin primes was offered.

\section{References}

[1] A Prime Number. Wikipedia. https://ru.wikipedia.org/wiki/Простое число\#cite note-27

[2] Baibekov, S.N. (2016) About Infinity of Prime Twins. Poisk, No, 203-213.

[3] Baibekov, S.N. (2015) Development of New Method for Generating Prime Numbers, Eurasian Gumilev University Bulletin, No. 4, 14-21.

[4] Baibekov, S.N. and Altynbek, S.A. (2015) Development of New Method for Generating Prime Numbers. Natural Science, 7, 416-423.

[5] Baibekov, S.N. (2015) Generation and Test of Prime Numbers. Eurasian Gumilev University Bulletin, No. 6, Part 2, 263-365. 
[6] Siegel, C.L. (1935) Acta Arithmetica, v. 1, 83-86.

[7] Numbers of Arithmetic Progression. universal ru en.academic.ru/...копия

[8] Karatsuba, A.A. (1975) The Fundamentals of Analytical Number Theory. M., Chapter 9.

[9] Linnik, Yu.V. (1961) The Dispersion Method in Binary Additive Problems. L.: LGU Publishing.

[10] Linnik, Yu., Barban, M. and Tshudakov, N. (1964) On Prime Numbers in an Arithmetic Progression with a Prime-Power Difference. Acta Arithmetica, 9, 375-390.

[11] Gritsenko, S.A. and Dhevtsova, M.V. (2011) On Distribution of primes in Arithmetic Progressions with a Difference of Special Type. Scientific Bulletin, Mathematics and Physics Series,. No (100), n. 22, 17-37.

[12] German-Yevtushenko, M.S. (2013) About a Sum of Values of Divisor Function by Numbers Lying in Arithmetic Progressions with a Difference of Special Type. Scientific Bulletin, Mathematics and Physics Series,. No 12(155), n. 31, 12-19.

[13] Zagier, D. (1977) The First 50 Million Prime Numbers. http://ega-math.narod.ru/Liv/Zagier.htm

Submit or recommend next manuscript to SCIRP and we will provide best service for you:

Accepting pre-submission inquiries through Email, Facebook, LinkedIn, Twitter, etc. A wide selection of journals (inclusive of 9 subjects, more than 200 journals)

Providing 24-hour high-quality service

User-friendly online submission system

Fair and swift peer-review system

Efficient typesetting and proofreading procedure

Display of the result of downloads and visits, as well as the number of cited articles Maximum dissemination of your research work

Submit your manuscript at: http://papersubmission.scirp.org/

Or contact apm@scirp.org 